\title{
Studying Neutral Current Elastic Scattering and the Strange Axial Form Factor in MicroBooNE
}

\author{
Katherine Woodruff for the MicroBooNE Collaboration* \\ New Mexico State University \\ E-mail: kwoodruf@nmsu.edu
}

\begin{abstract}
One of the least constrained contributions to the Neutral Current (NC) elastic neutrino-proton cross section is the strange axial form factor, which represents the strange quark spin contribution to the spin structure of the proton. This becomes the net strange spin contribution, $\Delta s$, in the limit when the negative four-momentum transfer squared $\left(Q^{2}\right)$ is zero. The strange axial form factor can be determined by studying NC elastic scattering events in the MicroBooNE detector. MicroBooNE's unique ability to detect low-energy protons is expected to allow the reconstruction of these events with a $Q^{2}$ as low as $0.10 \mathrm{GeV}^{2}$ and to determine the strange axial form factor in a model-independent approach. We present a selection of neutral current elastic events in a subset of MicroBooNE neutrino data, as well as our plan to extract the strange part of the axial form factor and $\Delta s$ from this selection in the full data set.
\end{abstract}

23rd International Spin Physics Symposium - SPIN2018 -

10-14 September, 2018

Ferrara, Italy

\footnotetext{
*Speaker.
} 


\section{Introduction}

The structure of a nucleon is more interesting than the three familiar up and down valence quarks. These three quarks only account for a small percent of the nucleon mass. The gluons that bind the quarks split into quark-antiquark pairs of up, down, and strange flavor. The remainder of the nucleon mass is carried by this quark-gluon sea. The structure of the sea and how its elements combine with the valence quarks to give the nucleon its measured structure are not precisely known.

The net spin of the proton comes from a combination of the spin and orbital momentum of the quarks and gluons. The net contribution from the spin of strange quarks and antiquarks, $\Delta s$, is defined as

$$
\begin{gathered}
\Delta s=\int_{0}^{1} \Delta s(x) d x \\
\Delta s(x)=\sum_{r= \pm 1} r\left[s^{(r)}(x)+\bar{s}^{(r)}(x)\right],
\end{gathered}
$$

where $s(\bar{s})$ is the spin-dependent parton distribution function of the strange (anti)quark, $r$ is the helicity of the quark relative to the proton helicity and $x$ is the Bjorken scaling variable [1]. In the static quark model this value is zero.

In the 1980s the European Muon Collaboration [2] and several subsequent experiments found that the Ellis-Jaffe Sum Rule was violated in polarized, charged-lepton, inclusive, Deep Inelastic Scattering (DIS). The Ellis-Jaffe sum rule [3] assumes that SU(3) flavor symmetry is valid and that $\Delta s=0$. For the results to be consistent with exact $\mathrm{SU}(3)$ flavor symmetry, $\Delta s$ must be negative. Follow-up measurements using charged-lepton semi-inclusive deep inelastic scattering have been consistent with $\Delta s=0$, but these determinations of $\Delta s$ are highly dependent on the fragmentation functions used [4].

An independent determination of $\Delta s$ can be made using Neutral-Current (NC) elastic neutrinoproton scattering. The NC elastic cross section depends directly on $\Delta s$ and no assumptions about $\mathrm{SU}(3)$ flavor symmetry or fragmentation functions are needed.

\subsection{Neutral Current Elastic Neutrino-Proton Scattering $(v p \rightarrow v p)$}

To see how the dependence on $\Delta s$ arises, it is illustrative to look at the simplest case of free nucleon scattering. In reality, the neutrinos are interacting with nucleons in an argon nucleus which can modify the scattering cross section and the observed final state. The Neutral-Current Elastic (NCE) neutrino-proton cross section for free nucleon scattering is given by [1],

$$
\begin{aligned}
\left(\frac{d \sigma}{d Q^{2}}\right)_{v}^{N C} & =\frac{G_{F}^{2}}{2 \pi}\left[\frac{1}{2} y^{2}\left(G_{M}^{N C}\right)^{2}+\left(1-y-\frac{M}{2 E} y\right) \frac{\left(G_{E}^{N C}\right)^{2}+\frac{E}{2 M} y\left(G_{M}^{N C}\right)^{2}}{1+\frac{E}{2 M} y}\right. \\
& \left.+\left(\frac{1}{2} y^{2}+1-y+\frac{M}{2 E} y\right)\left(G_{A}^{N C}\right)^{2} \pm 2 y\left(1-\frac{1}{2} y\right) G_{M}^{N C} G_{A}^{N C}\right]
\end{aligned}
$$

where $G_{A}^{N C}$ is the neutral-current axial form factor, $G_{E}^{N C}$ is the neutral-current electromagnetic form factor, and $G_{M}^{N C}$ is the neutral-current magnetic form factor. These form factors represent the finite structure of the proton. The axial form factor represents the spin structure of the proton. Individual quark contributions to $G_{E}$ and $G_{M}$ have been measured in electron-nucleon scattering, from which 
we can predict $G_{M}^{N C}$ and $G_{E}^{N C}$. The strange quark contribution to the electromagnetic form factors has been determined to be small. This allows us to determine $G_{A}^{N C}$ from NCE interactions.

To determine the net strange quark spin contribution we can write $G_{A}^{N C}$ in terms of quark flavor and extrapolate to $Q^{2}=0$ :

$$
\begin{aligned}
G_{A}^{N C}\left(Q^{2}\right) & =\frac{1}{2} G_{A}^{C C}\left(Q^{2}\right)+\frac{1}{2} G_{A}^{s}\left(Q^{2}\right), \\
G_{A}^{N C}\left(Q^{2}=0\right) & =\frac{1}{2}(\Delta u-\Delta d)-\frac{1}{2} \Delta s,
\end{aligned}
$$

where $(\Delta u-\Delta d)=g_{A}$ is the weak coupling constant which has been measured in neutron decay, and $G_{A}^{C C}$ is the charged-current axial form factor which contains information about the up and down quark spin distributions.

While nuclear effects can modify the final state, the signal of a NCE proton interaction is ideally a single proton track. Without a vertex to help identify the track as coming from a neutrino interaction, they are difficult to reconstruct. Everything that we know about this interaction, including momentum transfer, comes from the single proton track. Since we want to extrapolate $G_{A}^{N C}\left(Q^{2}\right)$ to zero, we need to be able to reconstruct very low energy protons. We estimate that we will be able to detect a proton track if it traverses at least $2.5 \mathrm{~cm}$ ( $\sim 8$ wires) in MicroBooNE. This is the range of a $50 \mathrm{MeV}$ kinetic energy proton in liquid argon, which corresponds to a $\mathrm{NC}$ elastic interaction with $Q^{2} \sim 0.10 \mathrm{GeV}^{2}$.

\section{Model}

The model of neutrino interactions in the MicroBooNE detector, including the expected neutrino flux, the neutrino-nucleon cross section, the effect of the nucleon in an argon nucleus, and the detector response, is simulated in the MicroBooNE software. The initial neutrino-argon interactions are simulated using the GENIE neutrino generator [5], and the final state particles are propagated through the detector geometry using Geant4 [6]. In the model, only the neutrino-nucleon cross section is affected by the strange axial form factor. The GENIE neutrino generator treats the nuclear effects and final state interactions as multiplicative factors which modify the neutrinonucleon cross section. This allows us to determine how the simulation would change due to a change in the neutrino-nucleon cross section by calculating the ratio of the new cross section to the cross section used in the simulation. This method is referred to as reweighting.

Even though the "signal" that we are optimizing for is NC elastic neutrino-proton interactions in the active detector, other interactions are affected by the strange axial form factor and the form of the elastic cross section in general. The cross sections for background NC elastic neutrino-neutron interactions and any NC elastic interactions that occur outside of the TPC are affected by the new strange form factor and need to be calculated. Charged current background events are not affected by the strange axial form factor, but we calculate new Charged Current Quasi-Elastic (CCQE) cross sections for these events so that the cross section parameterization and form factor models match the neutral current models that we are using. 


\subsection{Cross Section Model}

For reweighting both the NC elastic and CCQE cross sections, we use the Llewellyn-Smith form:

$$
\frac{d \sigma}{d Q^{2}}=\frac{G_{F}^{2}}{2 \pi E_{v}^{2}}\left[A \pm B W+C W^{2}\right]
$$

where the $+(-)$ is for (anti)neutrino scattering, $G_{F}$ is the Fermi coupling constant, $E_{v}$ is the incoming neutrino energy, $W=\frac{4 E_{\mathrm{v}}}{M_{p}}-\tau, \tau=\frac{Q^{2}}{4 M_{p}^{2}}$, and

$$
\begin{aligned}
A & =\frac{\left(m_{l}^{2}+Q^{2}\right)}{4}\left[G_{A}(1+\tau)-\left(F_{1}^{2}-\tau F_{2}^{2}\right)(1-\tau)+4 \tau F_{1} F_{2}\right], \\
B & =-\frac{Q^{2}}{4} G_{A}\left(F_{1}+F_{2}\right), \\
C & =\frac{Q^{2}}{64 \tau}\left[G_{A}^{2}+F_{1}^{2}+\tau F_{2}^{2}\right] .
\end{aligned}
$$

Here $m_{l}$ is the outgoing lepton mass and $G_{A}, F_{1}$, and $F_{2}$ are the axial, Dirac, and Pauli form factors, respectively. The form factors are different for $\mathrm{NC}$ and $\mathrm{CC}$ elastic scattering, with

$$
\begin{aligned}
F_{1,2}^{N C} & =\frac{1}{2} F_{1,2}^{C C}, \\
G_{A}^{N C} & =\frac{1}{2} G_{A}^{C C}+\frac{1}{2} G_{A}^{S},
\end{aligned}
$$

where $G_{A}^{s}$ is the strange quark contribution to the axial form factor.

\subsection{Form Factor Model}

For the form factor models, we use a $z$ expansion parameterization for all three of the form factors described in [7] and [8]. These parameterizations are made by mapping the negative fourmomentum transfer squared, $Q^{2}$, onto a domain where the strange axial form factor is analytic. This gives a new variable

$$
z\left(Q^{2}, t_{\text {cut }}, t_{0}\right)=\frac{\sqrt{t_{\text {cut }}+Q^{2}}-\sqrt{t_{\text {cut }}-t_{0}}}{\sqrt{t_{\text {cut }}+Q^{2}}+\sqrt{t_{\text {cut }}-t_{0}}},
$$

where $t_{\text {cut }}$ is the first pole and $t_{0}<t_{\text {cut }}$ is an arbitrary number which can be optimized for the $Q^{2}$ range of the data. The new variable, $z$, is guaranteed to be small with the optimal choice of $t_{0}$, so a Taylor expansion around it should converge. The form factors are defined by the Taylor expansion as

$$
G\left(Q^{2}\right)=\sum_{k=0}^{k_{\max }} a_{k} z\left(Q^{2}\right)^{k},
$$

where $a_{k}$ are the coefficients that are fit to data.

The fits that we use for the Dirac and Fermi form factors are in [7], and the fit for the chargedcurrent axial form factor is in [8].

We can use the same method to determine the strange part of the neutral-current axial form factor from the number of NC elastic events in MicroBooNE. We use $t_{c u t}=4 m_{\pi}$, the four-pion 
threshold which is the first pole in the axial form factor. If we set $k_{\max }=2$, this becomes a simple three parameter fit to the data

$$
G_{A}^{s}\left(Q^{2}\right)=a_{0}^{s}+a_{1}^{s} z\left(Q^{2}\right)+a_{2}^{s} z\left(Q^{2}\right)^{2} .
$$

We can redefine the strange axial mass, $M_{A}^{s}$, in terms of the slope of $G_{A}^{s}\left(Q^{2}\right)$ at $Q^{2}=0$ as it is in the dipole form

$$
M_{A}^{s} \equiv \sqrt{\frac{2 G_{A}^{s}(0)}{\left(G_{A}^{s}\right)^{\prime}(0)}}=\sqrt{\frac{2 a_{0}^{s}}{a_{1}^{s}}} .
$$

Since we also know that $G_{A}^{s}\left(Q^{2}=0\right) \equiv \Delta s$, we can write the first two coefficients in terms of physical parameters

$$
a_{0}^{s}=\Delta s, \quad a_{1}^{s}=\frac{2 \Delta s}{\left(M_{A}^{s}\right)^{2}} .
$$

\subsection{Proton track identification}

This section has been adapted from Ref. [9].

\subsubsection{Gradient decision tree boosting}

To identify proton tracks, we use a gradient-boosted decision tree classifier. We chose to use decision trees because they are easily interpretable and the inputs can be a mix of numeric and categorical variables. Below is a short description of gradient tree boosting. A more detailed description can be found in the documentation for the XGBoost[10] software library that was used.

A decision tree can be thought of as a series of if/else statements that separate a data set into two or more classes. The goal of each cut is to increase the information gain. For numerical variables any cut value can be selected by the tree. At each node of the tree, a split is chosen to maximize information gain until a set level of separation is reached. At the terminus of the series of splits, called a leaf, a class is assigned.

Two weaknesses of decision trees are their tendency to over fit the training data and the fact that the output is a class label and not a probability. Gradient-boosting addresses both of these issues by combining many weak classifiers into a strong one. Each weak classifier is built based on the error of the previous one. For a given training set, whenever a sample is classified incorrectly by a tree, that sample is given a higher importance when the next tree is being created. Mathematically, each tree is training on the gradient of the loss function. After all of the trees have been created, each tree is given a weight based on its ability to classify the training set, and the output of the gradient-boosted decision tree classifier is the probability that a sample is in a given class.

\subsubsection{The decision tree model}

We created a multi-class gradient-boosted decision tree classifier, using the XGBoost software library, to separate five different track types: any proton track, muons or pions from Booster Neutrino Beam (BNB) neutrino interactions, tracks from electromagnetic showers from BNB interactions, and any non-proton track produced by a cosmic ray interaction. The classifier takes reconstructed track features as input and outputs a probability of the track having been produced 
by each of the given particle types. The reconstructed features are based on the track's geometric, calorimetric, and optical properties from MicroBooNE charge and light collection systems.

The training data that we use to make the decision trees comes from Monte Carlo simulation. The BNB interactions are simulated using the GENIE neutrino generator, and cosmic interactions are simulated using the CORSIKA cosmic ray generator [11]. The particles generated by GENIE and CORSIKA are passed to Geant 4 where they are propagated through a simulated MicroBooNE detector. For training and testing of the trees we only use tracks that were reconstructed in LArSoft.

Fig. 1 shows the decision tree proton score for all tracks that are contained in the MicroBooNE detector for both a subset of the neutrino beam data corresponding to $5 \times 10^{19}$ Protons On Target (POT) and the Monte Carlo simulation.
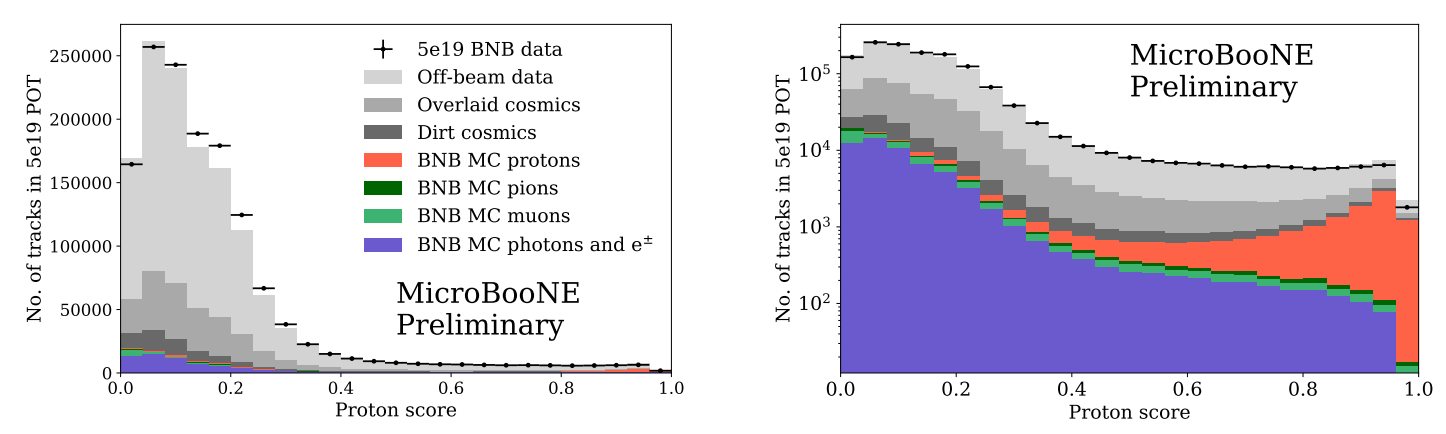

Figure 1: Data to Monte Carlo comparisons of proton ID scores. The left plot shows linear scale, and the right plot shows the same thing in a log scale. The gray filled histograms are cosmic background tracks, the color filled histograms show neutrino-induced Monte Carlo tracks, and the black points show tracks in $5 \times 10^{19}$ POT data. The dashed line shows the proton ID cut for the NC elastic pre-selection. Uncertainty bars are statistical only.

If we keep only tracks that have a proton score greater than 0.5 (anything more likely than not to be a proton) we have an overall proton identification efficiency of approximately $80 \%$. This does not include the reconstruction efficiency for protons.

Fig. 2 shows the efficiency of protons from simulated neutral current elastic events with a proton score greater than 0.5 . Both the proton identification efficiency and the overall, reconstruction plus identification, efficiency as a function of true proton kinetic energy are shown for NC elastic events. The average proton ID efficiency over all energies is $75 \%$ for NC elastic proton interactions. When including the efficiency of track reconstruction, the average overall proton efficiency for NC elastic events is $60 \%$. The proton kinetic energy range of interest for this analysis is from 0.05 to $0.5 \mathrm{GeV}$, which end at the very beginning of the drop in proton efficiency. The average proton ID efficiency in the $0.05-0.5 \mathrm{GeV}$ kinetic energy range is $80 \%$ for NC elastic protons and $64 \%$ when including the efficiency of track reconstruction. 

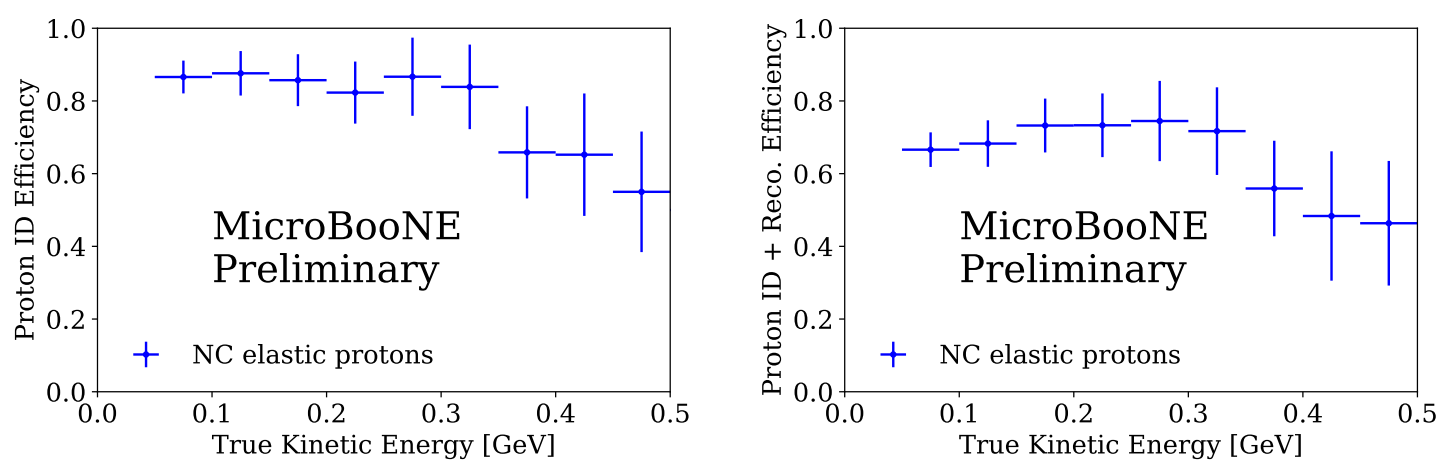

Figure 2: The proton efficiency in neutral current elastic interactions. The left plot shows the NC elastic proton identification efficiency as a function of true proton kinetic energy. The proton identification efficiency is defined as the fraction of reconstructed true proton tracks that are correctly identified as protons. The right plots shows the overall NC elastic proton efficiency as a function of true proton kinetic energy. The overall proton efficiency is defined at the fraction of simulated NC elastic protons that are reconstructed and correctly identified at protons. The decrease in efficiency at larger proton kinetic energy is due to the increased probability of the proton reinteracting in the argon. The uncertainty is statistical only.

\section{Selection}

We start the selection by making pre-cuts to remove a large amount of the background. The remaining events are evaluated using a logistic regression model to determine how signal-like they are (described in Sec. 3.2). The logistic regression output gives us a parameter to tune to maximize our ability to extract the strange axial form factor parameters.

\subsection{Pre-Selection}

To reduce the cosmic background, we rejected any events that did not have a reconstructed flash with at least 6.5 photoelectrons within the $1.6 \mu$ s beam timing window. We select reconstructed tracks that were fully contained in the TPC fiducial volume and at least $2.5 \mathrm{~cm}$ long as potential proton candidates. The track is selected if it is classified as a proton with a greater than $50 \%$ probability by the gradient-boosted decision tree classifier described in Sec. 2.3.

\subsection{Logistic Regression of Selection Variables}

Our NC elastic events of interest appear as a single isolated proton that produces scintillation light during the beam-spill window. The reconstructed variables that we use to select these events are: (1) the decision tree proton ID score, (2) the distance from the track to the reconstructed flash in the $z$ direction, (3) the distance from the track to the reconstructed flash in the $y$ direction, (4) the distance between the track candidate and the next closest reconstructed track, (5) whether or not the track is in the beam direction, (6) the distance between any reconstructed tracks IDed as neutrinoinduced muons and the reconstructed beam flash, and (7) the distance between any reconstructed tracks IDed as neutrino-induced pions and the reconstructed beam flash.

The proton ID score is the same as used in the pre-selection and described in detail in Sec. 2.3. The muon and pion background candidates are defined as any reconstructed track that has a muon 
ID or pion ID score greater than 0.5 from the boosted decision tree classifier. We define the onedimensional distances between reconstructed tracks and reconstructed flashes as the difference in position between the midpoint of the track (defined by its endpoints) and the photoelectronweighted center of the flash.

To determine which events are NC elastic like based on these seven variables, we use them as input to a logistic regression model [12]. In logistic regression we fit a multi-dimensional sigmoid function to the signal and background data. The output is a score that can be used to determine how signal-like a data point is:

$$
S(g(\mathbf{x}))=\frac{e^{g(\mathbf{x})}}{1+e^{g(\mathbf{x})}},
$$

where $g(\mathbf{x})$ is a linear combination of the selection variables, $\mathbf{x}$ :

$$
g(\mathbf{x})=w_{0}+w_{1} x_{1}+w_{2} x_{2}+\ldots+w_{7} x_{7} .
$$

Here $x_{1}$ is the proton ID score, $x_{2}$ is the distance to the flash, etc. The set of weights, $w_{0}, \ldots, w_{7}$ are determined from a fit to the data. We determined these weights using the StatsModels module [13] in Python to fit the model to a purely Monte Carlo data set.

If we choose a score cut of 0.8 , for example, we get an overall signal efficiency of $19 \%$ and purity of $18 \%$. Fig. 3 shows the signal selection efficiency as a function of true negative fourmomentum transfer for a 0.8 score cut. The reconstructed four-momentum transfer is determined entirely from the proton kinetic energy using

$$
\begin{aligned}
Q_{p}^{2} & =-q^{2}=-\left(\mathbf{p}_{\mathbf{p}}^{\prime}-\mathbf{p}_{\mathbf{p}}\right)^{\mathbf{2}} \\
& =-\left(E_{p}^{\prime}-E_{p}\right)^{2}+\left(\bar{p}_{p}^{\prime}-\bar{p}_{p}\right)^{2} \\
& =2 T_{p} M_{p},
\end{aligned}
$$

where $\mathbf{p}$ is four-momentum, $E$ is energy, $\bar{p}$ is three-momentum, $M$ is mass, $T$ is kinetic energy determined by the length of the track, the $p$ subscript represents the proton in the neutrino-proton interaction, the prime represents the final state, and the proton momentum in the nucleus is assumed to be small compared to the final proton momentum. When the final proton momentum is close to the initial proton momentum in low $Q^{2}$ interactions, this assumption is not as strong and we rely more heavily on the nuclear model in the GENIE simulation.

Figs. 4, 5, and 6 show the signal and backgrounds selected compared to data for a 0.8 logistic regression score cut. The number of simulated events and off-beam data events have been normalized to the $5 \times 10^{19}$ POT of on-beam data.

\subsection{Remaining Backgrounds}

The remaining backgrounds fall into four separate categories: (1) neutral current back interactions in the TPC, (2) charged current interactions in the TPC, (3) neutrino interactions outside of the TPC, and (4) cosmic interactions in time with the beam.

The first category, NC backgrounds in the TPC, include NC elastic interactions with a neutron (35\%), NC elastic interactions with a correlated neutron-proton pair (35\%), NC resonant interactions ( $25 \%$ ), and NC DIS interactions (4\%). The last two, resonant and DIS, are mainly due to 

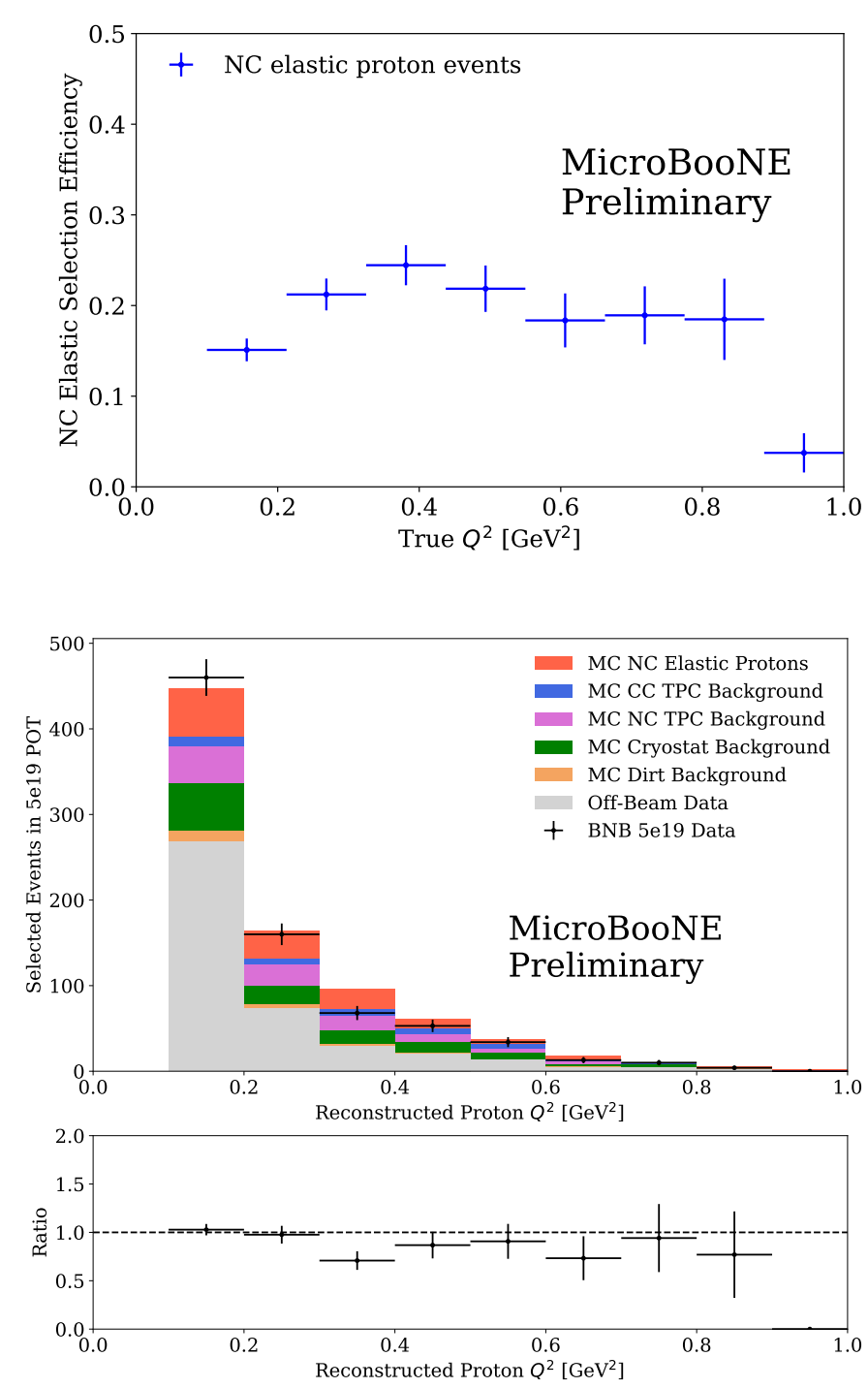

Figure 3: Neutral current elastic selection efficiency as a function of true fourmomentum transfer for a logistic regression score cut of 0.8 . The uncertainties are statistical only.

Figure 4: NC elastic event selection given a logistic regression score cut of 0.8 as a function of reconstructed negative fourmomentum transfer. The top plot shows the signal and itemized backgrounds compared to the BNB $5 \times 10^{19}$ POT data in black. The bottom plot shows the ratio of BNB $5 \times 10^{19}$ POT data to BNB MC with cosmic overlay and off-beam data. In both plots the uncertainties are statistical only.

tracks being mis-reconstructed or not reconstructed at all. The first two are due to the fact that neutrons are very difficult to detect in LArTPCs. In the case of the NC elastic neutron interactions, we select events in which the neutron scatters with a proton mimicking a NC elastic neutrino-proton event. In the case of NC interactions with correlated neutron-proton pairs, we select the proton from the initial interaction, but the neutron goes undetected.

The second category, CC interactions in the TPC, are almost entirely from the muon track being mis-reconstructed, mis-identified as a cosmic, or not reconstructed at all. In these events, a secondary proton from the interaction or a coincident cosmic proton can be selected as a neutrinoinduced proton.

The third category, TPC-external interactions, occur when a neutrino from the beam interacts outside of the active TPC volume. A neutron from this interaction can enter the TPC and elastically scatter with a proton. The neutrino interaction occurs in the liquid argon inside of the cryostat $\sim 70 \%$ of the time, and outside of the cryostat in the dirt upstream of the detector hall the other $\sim 30 \%$ of the time. These cryostat-external, "dirt", interactions are simulated separately from the 

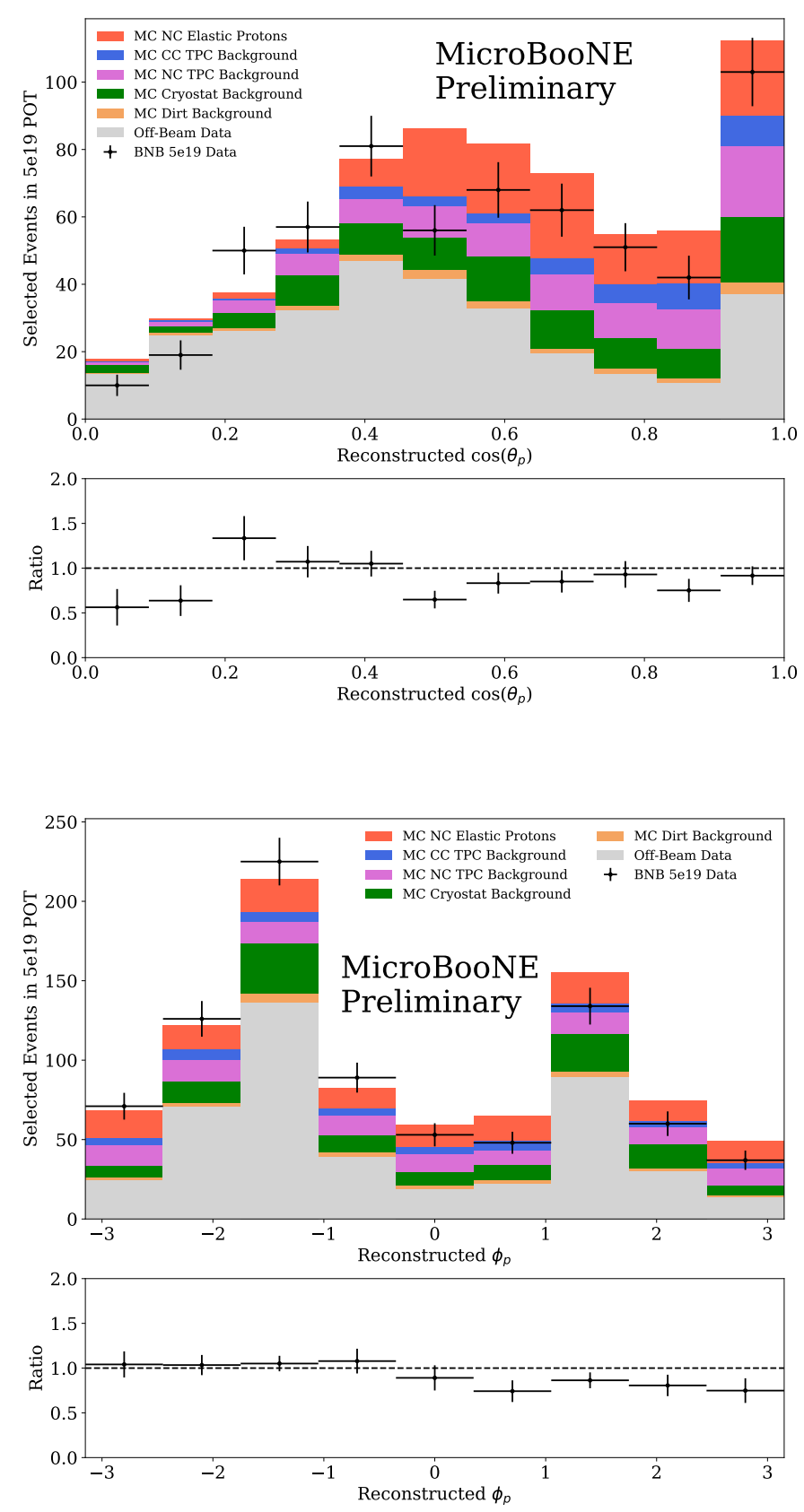

Figure 5: NC elastic event selection given a logistic regression score cut of 0.8 as a function of reconstructed proton $\cos \left(\theta_{p}\right)$, which is the proton angle with respect to the neutrino beam direction. The top plot shows the signal and itemized backgrounds compared to the BNB $5 \times 10^{19}$ POT data in black. The bottom plot shows the ratio of BNB $5 \times 10^{19}$ POT data to BNB MC with cosmic overlay and offbeam data. In both plots the uncertainties are statistical only.

Figure 6: $\mathrm{NC}$ elastic event selection given a logistic regression score cut of 0.8 as a function of reconstructed proton $\phi$. The top plot shows the signal and itemized backgrounds compared to the BNB $5 \times 10^{19}$ POT data in black. The bottom plot shows the ratio of BNB $5 \times 10^{19}$ POT data to BNB MC with cosmic overlay and off-beam data. In both plots the uncertainties are statistical only.

interactions inside the cryostat. The normalization of this dirt sample was determined by fitting the flash position in the beam direction to data. There is an observed excess of flashes from dirt events in the upstream end of the detector. This region is excluded in the analysis with fiducial cuts.

The last category, cosmics in time with the beam, is also the largest. However, we can determine the rate and distribution of this background very well by taking data while the neutrino beam is not on. This is the "off-beam" data set. These events can then be subtracted from the "on-beam" data. 


\section{Conclusions}

The contribution of the strange quark spin to the spin of the proton is an open and interesting question. A lot can be learned about this physics through the strange part of the axial form factor, including the net strange spin contribution, $\Delta s$, which is simply the value of the strange axial form factor, $G_{A}^{s}$, at zero four-momentum transfer. MicroBooNE's ability to detect low-energy protons translates into an ability to measure $G_{A}^{s}$ at low four-momentum transfer.

Selecting isolated proton-like reconstructed tracks near a beam flash gives us sets of events with an enhanced fraction of NC elastic events. The efficiency and purity of this selection, as well as the agreement between the selection in Monte Carlo and the selection in the BNB $5 \times 10^{19}$ POT data set can be tuned to optimize the sensitivity to $\Delta s$ using the output of our logistic regression model.

Assuming the current level of neutral current elastic proton event selection efficiency, we expect to select on the order of $1000 \mathrm{NC}$ elastic events in the full MicroBooNE data set with a $Q^{2}$ down to $0.1 \mathrm{GeV}^{2}$. The next stage of this analysis will include updated and improved energy calibration which is expected to increase the agreement between data and simulation. A full treatment of the systematic uncertainty due to the physics models, the proton selection, and the detector efficiency will also be included. This should allow us to extract the strange axial form factor parameters with a greater precision than has previously been possible in neutrino-nucleon scattering experiments.

\section{References}

[1] W. M. Alberico, Samoil M. Bilenky, and C. Maieron. Phys. Rept., 358 (2002) 227-308.

[2] J. Ashman et al. Nucl. Phys. B, 328 (1989) 1.

[3] John Ellis and Robert Jaffe. Phys. Rev. D, 9 (1974) 1444-1446.

[4] Christine A. Aidala, Steven D. Bass, Delia Hasch, and Gerhard K. Mallot. Rev. Mod. Phys., 85 (2013) 655-691.

[5] C. Andreopoulos et al. Nucl. Instrum. Meth. A, 614 (2010) 87-104.

[6] S. Agostinelli et al. Nucl. Instrum. Meth. A, 506 (2003) 250-303.

[7] Zhihong Ye, John Arrington, Richard J. Hill, and Gabriel Lee. Phys. Lett. B, 777 (2018) 8-15.

[8] Aaron S. Meyer, Minerba Betancourt, Richard Gran, and Richard J. Hill. Phys. Rev. D, 93 (2016) 11.

[9] The MicroBooNE Collaboration. MicroBooNE Public Note, 1025 (2017).

[10] Tianqi Chen and Carlos Guestrin. CoRR (2016).

[11] D. Heck, G. Schatz, T. Thouw, J. Knapp, and J. N. Capdevielle. (1998).

[12] David W. Hosmer and Stanley Lemeshow. Wiley (2005).

[13] Skipper Seabold and Josef Perktold. 9th Python in Science Conference, Austin, Texas (2010). 